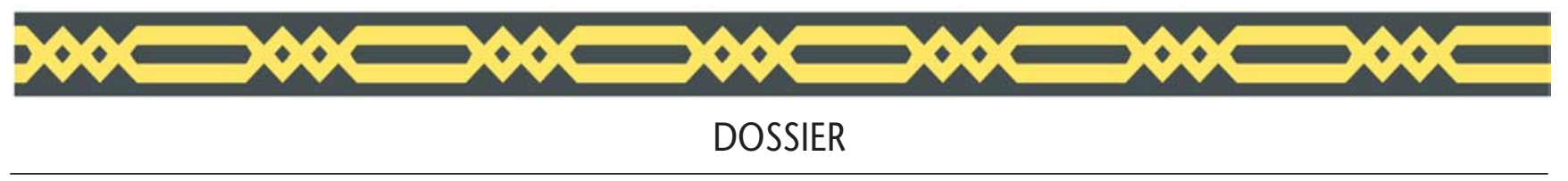

\title{
Le DHA dans la neurotransmission
}

Monique LAVIALLE

Gaëlle CHAMPEIL-POTOKAR

Isabelle DENIS

Philippe GUESNET

Fabien PIFFERI

Sylvie VANCASSEL

Unité de Nutrition et régulation Lipidique

des fonctions Cérébrales,

INRA, Jouy en Josas, 78352, France.

Fax : 0134652311

<Monique.Lavialle@jouy.inra.fr>

\section{Introduction}

Depuis une dizaine d'années, les études démontrant l'implication des acides gras polyinsaturés (AGPI) de la série oméga 3 ou n-3 et notamment de l'acide docosahexaénoïque (DHA) dans nombre de manifestations comportementales et neurophysiologiques justifient de rechercher plus précisément les points d'impact du DHA au cœur d'une «machinerie » particulièrement complexe : la synapse. C'est en effet le point clé de la transmission de l'influx entre les neurones, et de la propagation de l'information dans le cerveau. L'organisation et la structuration des réseaux neuronaux s'établissent majoritairement pendant la période périnatale. Le cerveau adulte n'est pas exempt de synaptogenèse (croissance neuritique, établissement de nouvelles synapses) en particulier dans les processus de mémorisation et d'apprentissage. Cependant la synapse établie n'est pas figée. L'efficacité de la neurotransmission dépend du microenvironnement et met en jeu des mécanismes et des facteurs multiples potentiellement modulables par le DHA.

Les effets observés de la déficience membranaire en DHA sur différents systèmes de neurotransmission (monoaminergique, cholinergique et glutamatergique) rendent compte de modifications de la libération du neurotransmetteur dans différentes structures. Cependant la disparité des réponses en fonction du neurotransmetteur étudié et des zones cérébrales concernées ne permet pas pour l'instant d'envisager une piste commune à ces perturbations.

\begin{abstract}
Long chain polyunsaturated fatty acids (PUFAs), particularly arachidonic acid and docosahexaenoic acid (DHA), are integral components of neural membrane phospholipids. DHA deficiency is associated with behavioural and neurophysiological disorders. A deficiency of DHA markedly affects neurotransmission, membrane-bound proteins, ion channel activities and synaptic plasticity, and the supplementation restores neurotransmission. Although the molecular mechanism of DHA involvement remains unknown, more and more data demonstrate its implication in various cellular activities contributing to regulation of neurotransmission. Since recent studies have provided evidence that $n-3$ deficiency altered neurogenesis in embryonic brain, the question of lasting effects on neural function can be addressed.
\end{abstract}

Key words: polyunsatured fatty acid, neurotransmitter, monoamine, acetylcholine, synapse, DHA

L'étendue de l'action des AGPI en tant qu'éléments structurants des membranes, seconds messagers lipidiques et régulateurs de gènes, laisse entrevoir plusieurs cibles possibles.

Après un rapide exposé des données expérimentales, nous élargirons la question à l'ensemble de l'environnement synaptique et présenterons les perspectives de réflexions à la lumière des recherches d'actualité mettant en exergue l'aspect dynamique de la synapse qui est un des éléments de la plasticité cérébrale.

\section{Le DHA, I'AGPI des membranes cérébrales}

Le système nerveux central possède la plus forte concentration en lipides après le tissu adipeux, soit $30 \%$ du poids sec du cerveau. Les acides gras polyinsaturés (AGPI) à longue chaîne, particulièrement l'acide arachidonique (20:4n-6, AA) et l'acide docosahexaénoïque (22: $6 n-3, D H A)$, sont des composants fondamentaux des phospholipides membranaires des cellules neurales. Le taux élevé de DHA notamment dans la phosphatidyléthanolamine $(\mathrm{PE})>20 \%$ dans le cerveau et $>40 \%$ dans la rétine, caractérise le tissu nerveux. Au niveau subcellulaire, les taux les plus élevés se trouvent dans les membranes des synaptosomes.

Les précurseurs métaboliques respectifs des deux familles d'AGPI, à savoir l'acide linoléique (18: $2 n-6)$ pour la famille $n-6$ et l'acide a-linolénique $(18: 3 n-3)$ pour la famille $n-3$, sont des acides gras essentiels pour l'homme qui ne peut les synthétiser de novo. On les trouve principalement dans les graines et les huiles végétales. Après leur absorption, ces deux acides gras conduisent à la synthèse spécifique de dérivés actifs à plus longue chaîne I'AA pour la famille $n-6$, et les acides eicosapentaénoïque (20:5n-3, EPA) et DHA pour la famille $n-3$.

La teneur en AGPI n-6 et n-3 dans la composition membranaire est fonction de l'apport alimentaire en précurseurs ou en dérivés à plus longue chaîne.

L'équilibre entre $A A$ et DHA constitue un déterminant majeur des processus d'acquisition et de maturation des fonctions cérébrales [1]. C'est durant la période périnatale que la qualité des apports en AGPI semble déterminante pour le développement du cerveau, notamment au moment de la migration neuronale, de la myélinisation, de la croissance neuritique, et de la synaptogenèse [2]. Chez l'animal, une carence alimentaire profonde en AGPI n-3 expérimentalement induite dès le début de l'embryogenèse, réduit considérablement la teneur membranaire en DHA compensée par I'apparition d'un acide gras de la série n-6 (l'acide docosapentaénoïque, 22 : 5n-6, DPA) perturbant fortement l'équilibre $n-6 / n-3$ [3].

Le tableau 1 illustre l'effet de la carence alimentaire et souligne certaines différences de composition entre les structures étudiées. Cette observation peut s'expliquer par l'organisation et la structure plus ou moins complexe spécifique à chacune.

L'impact de ce remaniement membranaire sur la neurotransmission sera présenté en considérant les manifestations à différents niveaux, comportemental, physiologique et cellulaire. 
Tableau 1. Comparaison des taux membranaires des principaux AGPI dans différentes structures cérébrales de hamsters élevés avec soit un régime équilibré en AGPI n-6 et n-3 (témoins) soit un régime carencé en AGPI n-3 (déficients).

\begin{tabular}{|c|c|c|c|c|c|}
\hline & & Cortex frontal & $\begin{array}{c}\text { Noyau } \\
\text { suprachiasmatique }\end{array}$ & Striatum & Hippocampe \\
\hline \multirow[t]{4}{*}{ Témoins } & $\mathrm{AA}(20: 4 n-6)$ & $13,7 \pm 1,7$ & $15,9 \pm 0,7$ & $12,9 \pm 0,6$ & $13,3 \pm 0,5$ \\
\hline & $\operatorname{DPA}(22: 5 n-6)$ & $2,6 \pm 1,4$ & $0,4 \pm 0,1$ & $0,3 \pm 0,0$ & $0,6 \pm 0,1$ \\
\hline & $\mathrm{DHA}(22: 6 n-3)$ & $21,2 \pm 5,4^{a}$ & $16,0 \pm 1,0^{b}$ & $16,4 \pm 0,6^{b}$ & $18,8 \pm 0,7^{\mathrm{a}}$ \\
\hline & $n-6 / n-3$ & $0,9 \pm 0,1$ & $1,4 \pm 0,1$ & $1,1 \pm 0,0$ & $1,0 \pm 0,1$ \\
\hline \multirow[t]{4}{*}{ Déficients } & $\mathrm{AA}(20: 4 n-6)$ & $12,2 \pm 3,3^{a}$ & $19,8 \pm 0,8^{b *}$ & $15,0 \pm 1,8^{c}$ & $15,6 \pm 0,4^{c *}$ \\
\hline & $\operatorname{DPA}(22: 5 n-6)$ & $9,9 \pm 1,5^{\text {a* }}$ & $5,7 \pm 1,1^{b *}$ & $9,5 \pm 2,1$ a* & $9,6 \pm 1,2^{a *}$ \\
\hline & $\mathrm{DHA}(22: 6 n-3)$ & $9,4 \pm 2,1$ * & $9,7 \pm 0,8$ * & $7,6 \pm 0,5$ * & $8,7 \pm 0,8$ * \\
\hline & $n-6 / n-3$ & $2,9 \pm 0,3$ * & $3,4 \pm 0,3$ * & $4,0 \pm 0,2$ * & $3,6 \pm 0,3$ * \\
\hline
\end{tabular}

* différence significative $p<0,001$ pour chaque acide gras entre témoins et déficients dans chaque structure. $a, b, c$ différence significative $p<0,01$ entre les structures pour chaque acide gras dans chaque groupe.

\section{Troubles comportementaux}

Les études aujourd'hui très nombreuses convergent pour établir une relation entre un apport alimentaire déficient en n-3 et des perturbations comportementales.

Les données les plus fréquentes portent sur les tests cognitifs pour lesquels on évalue le nombre d'essais nécessaires pour que l'animal réponde positivement à un test d'évitement (shuttle box) [4], à un test de choix (discrimination olfactive) [5] ou à un test d'apprentissage (Piscine de Morris) [6-8]. Les performances chez les animaux déficients sont inférieures à celles des animaux contrôles illustrant une moindre efficacité de leur capacité d'apprentissage.

Dans un registre différent, il a été observé chez des rats déficients une augmentation de l'activité motrice mesurée par les temps et la longueur de déplacements dans la piscine de Morris [6, 7], les distances parcourues dans I'openfield $[9,10]$ et le nombre de déplacements latéralisés dans un couloir circulaire [11].

\section{Modulation de l'activité neuronale basale}

À un instant donné, un cerveau « au repos » présentera une image hétérogène découpée en zones plus ou moins actives en termes de consommation de glucose, de synthèse protéique ou de phosphorylation oxydative. L'étude de l'incidence d'une déficience en DHA nécessite d'intégrer cette disparité. Chez les rats déficients, la consommation de glucose et la phosphorylation oxydative sont réduites de $30 \%$ quelle que soit la zone cérébrale considérée (cortex frontal, hippocampe, noyau suprachiasmatique siège de l'horloge circadienne) [12]. Nous avons par ailleurs montré que cet effet était associé à une réduction de $30 \%$ la quantité des transporteurs de glucose au niveau de la barrière hématoencéphalique et au niveau des pieds astrocytaires [13].

\section{Modulation \\ de la neurotransmission par le DHA}

Les données acquises s'appuient sur la mesure du taux tissulaire ou du taux libéré de neurotransmetteurs par la technique de microdialyse. Dans certains cas, d'autres paramètres tels que les métabolites, le nombre de vésicules de stockage et les récepteurs post-synaptiques ont également été analysés. La majorité des travaux porte sur la neurotransmission monoaminergique dont les principaux sont reportés dans le tableau 2.

Les résultats apparemment divergents doivent être analysés en considérant l'espèce animale, la structure étudiée, l'âge et l'état de l'animal (anesthésié ou vigile), la durée de la carence alimentaire, l'heure de la journée à laquelle est fait le prélèvement, et la technique utilisée.

Certains points méritent plus particulièrement d'être soulignés. Si d'une façon générale, la carence en AGPI n-3 provoque une réduction du taux de dopamine et de sérotonine dans le cortex frontal du rat associé à une réduction du stock vésiculaire, il est à noter que cette tendance est inversée dans le noyau accumbens chez le rat vigile mais pas chez le rat anesthésié [14]. Par ailleurs, aucun effet n'est observé dans cette même structure chez le hamster, alors qu'une augmentation a pu être mesurée dans le striatum lorsque celui-ci a été prélevé $2 \mathrm{~h}$ avant la transition jour/nuit. Aucune modification n'a été mise en évidence $2 \mathrm{~h}$ après le début de la nuit lorsque le taux de dopamine est bas (données personnelles non publiées). À l'inverse, aucune modification n'a été mise en évidence dans le cortex frontal du hamster adulte.
Chez le rat nouveau-né dont la mère a reçu un régime enrichi en huile de poisson (forte en DHA et faible en EPA) $10 j$ avant l'accouplement, le taux de dopamine est significativement réduit comparativement à celui d'un rat issu d'une mère recevant un régime déficient en $n-3$ [16]. Ces résultats mettent en avant un effet antagonique de forte teneur en DHA sur la dopamine. Cependant, d'autres études $d^{\prime}$ 'apports enrichis en huile de poisson sur rat adulte mettent en évidence un effet inverse, à savoir l'augmentation de la dopamine et de la sérotonine dans le cortex frontal [19] alors que la teneur en AA était réduite et celle du DHA restait inchangée. Ces travaux sur la supplémentation apportent des résultats contradictoires qui pourraient s'expliquer en partie par la différence des rapports entre AA et DHA ou par le stade de développement.

Plusieurs équipes se sont intéressées à la « sensibilité de la période postnatale ». Ainsi, une étude chez le porcelet nourri avec des formules au $1^{\text {er }}$ jour de la naissance montre qu'une alimentation pauvre en AGPI ( $n-6$ et $n-3$ ) pendant les 18 premiers jours suffit pour abaisser la teneur membranaire en $\mathrm{AA}$ et $\mathrm{DHA}$, et diminuer le taux tissulaire de dopamine et de sérotonine dans le cortex frontal [20]. Des expériences sur la réversibilité de la déficience montrent qu'il est possible par une réalimentation équilibrée de rétablir la composition membranaire en DHA à condition qu'elle soit fournie avant l'âge du sevrage. Ce «rattrapage » ne permet cependant pas de récupérer la réponse dopaminergique [21]. Ces résultats sont à rapprocher de ceux de Moriguchi et al. [7] qui appliquant le même protocole confirment l'âge critique du sevrage pour pouvoir jouer sur la composition membranaire cérébrale et montrent par ailleurs que le retour à une réponse comportementale "normale » dépend aussi de la durée de la réalimentation.

Les études sur la neurotransmission cholinergique se résument à 4 études. L'une utilise des rats SHRSP (spontanément hypertendus) sur lesquels il a été démontré qu'un régime à $5 \%$ de DHA permet d'acquérir un taux d'acétylcholine (Ach) dans l'hippocampe comparable à celui de rats témoins.

Les autres s'appuient sur les mêmes protocoles de carence alimentaire que pour la dopamine, pour lesquelles il a été montré que chez des rats déficients en AGPn-3 la libération basale d'ACh est augmentée alors que la libération sous stimulation de $\mathrm{KCl}$ est réduite [22]. Des expériences de supplémentation en DHA sous forme d'huile de poisson permettent de rétablir la réponse pour des apports de $200 \mathrm{mg} / \mathrm{kg} / \mathrm{jour}$ [23]. L'observation originale d'une réduction du nombre de cellules immunoréactives choline-acetyl-transférase uniquement dans le striatum droit, nous a conduit à prospecter 
Tableau 2. Effet de la carence alimentaire en AGPIn-3 sur la neurotransmission monoaminergique

\begin{tabular}{|c|c|c|c|c|c|c|c|c|c|}
\hline \multirow[t]{2}{*}{ Modèles } & \multirow{2}{*}{$\begin{array}{l}N-3 \\
\text { Def }\end{array}$} & \multirow[t]{2}{*}{ Zones } & \multicolumn{3}{|c|}{ Dopamine libérée } & \multirow[t]{2}{*}{ DOPAC HVA } & \multirow[t]{2}{*}{ Récept } & \multirow{2}{*}{$\begin{array}{c}\text { Stock } \\
\text { Vésicules }\end{array}$} & \multirow[t]{2}{*}{ Réf } \\
\hline & & & Tissu & basale & activée & & & & \\
\hline $\begin{array}{l}\text { Rat } \\
\text { adulte } \\
\text { Anesthesié } \\
\text { Vigile (v) }\end{array}$ & $\mathrm{G} 2$ & $\begin{array}{c}\text { CxF } \\
\text { N Acc } \\
\text { Striatum } \\
\text { Cervelet } \\
\text { CA1 }\end{array}$ & $\begin{array}{l}\searrow \\
= \\
=\end{array}$ & 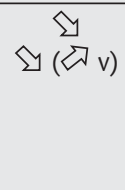 & $\stackrel{\searrow}{\unlhd}$ & 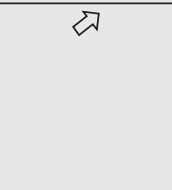 & $\begin{array}{l}\mathrm{D} 2 \unlhd \\
\pi\end{array}$ & $\stackrel{\searrow}{\unlhd}$ & $\begin{array}{c}\text { revue } \\
{[14]}\end{array}$ \\
\hline $\begin{array}{l}\text { Rat } \\
\text { Nv-Né }\end{array}$ & $\mathrm{G} 1$ & Cerveau & 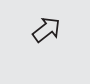 & & & $2 \pi$ & & & [16] \\
\hline $\begin{array}{l}\text { Hamster } \\
\text { adulte }\end{array}$ & G1 & $\begin{array}{c}\mathrm{CxF} \\
\text { Striatum }\end{array}$ & $\begin{array}{l}= \\
\unrhd\end{array}$ & & & $\begin{array}{l}= \\
2\end{array}$ & & & Non publié \\
\hline
\end{tabular}

\begin{tabular}{|c|c|c|c|c|c|c|c|c|}
\hline \multirow[t]{2}{*}{ Modèles } & \multirow{2}{*}{$\begin{array}{l}\mathrm{N}-3 \\
\text { Def }\end{array}$} & \multirow[t]{2}{*}{ Zones } & \multicolumn{3}{|c|}{ Sérotonine libérée } & \multirow[t]{2}{*}{ 5HIAA } & \multirow[t]{2}{*}{ Récept } & \multirow[t]{2}{*}{ Réf } \\
\hline & & & Tissu & basale & activée & & & \\
\hline $\begin{array}{l}\text { Rat } \\
\text { adulte } \\
\text { Anesthesié } \\
\text { Vigile (v) }\end{array}$ & G2 & $\begin{array}{c}\text { CxF } \\
\text { Striatum } \\
\text { Cervelet } \\
\text { CA1 }\end{array}$ & $\begin{array}{l}= \\
= \\
=\end{array}$ & 2 & $\unlhd$ & & $5 \mathrm{HT}^{2} \curvearrowright$ & {$[18]$} \\
\hline $\begin{array}{l}\text { Rat } \\
\text { Nv-Né }\end{array}$ & G1 & Cerveau & 2 & & & $=$ & & [16] \\
\hline $\begin{array}{l}\text { Hamster } \\
\text { adulte }\end{array}$ & G1 & $\begin{array}{c}\mathrm{CxF} \\
\text { Striatum }\end{array}$ & $\begin{array}{l}= \\
2 \nabla\end{array}$ & & & $\begin{array}{l}= \\
2 \nabla\end{array}$ & & Non publié \\
\hline
\end{tabular}

Colonne 1 : espèce et âge auquel l'observation a été faite

Colonne 2 : durée de la carence alimentaire (G1 = les mères sont carencées 15 j avant l'accouplement et la 1 ère portée donne les animaux expérimentaux ; G2 = 2 générations complètes de carence)

Colonne 3 : structures étudiées: CxF : cortex frontal ; N Acc : Noyau Accumbens; CA1 : couche de l'hippocampe

Colonne 4 : dosage sur tissu prélevé ou sur échantillon récupéré par microdialyse en situation basale ou stimulée sur animal anesthésié ou vigile (V)

Colonne 5 : dosage des métabolites (DOPAC : acide 3,4-dihydroxyphenylacetique; HVA : acide homovanillique ; 5HIAA : acide 5-hydroxyindoleacetique)

Colonne 6 : récepteurs

Colonne 7 : nombre de vésicules de stockage du neurotransmetteur ( $₫$ : augmentation, $\searrow$ : diminution ; : : pas d'effet).

I'incidence possible du DHA sur la latéralisation des animaux. Vancassel et al. [11] ont en effet démontré que la carence alimentaire en AGPI n-3 supprimait la préférence droitière observée chez les contrôles.

\section{Modulation de la synapse par le DHA}

Si le processus de la neurotransmission peut être schématisé simplement par l'excitation d'un neurone présynaptique, la libération d'un neurotransmetteur et l'activation de récepteurs post-synaptiques qui permettront la transmission et la propagation de l'influx, il n'est cependant pas représentatif des interrelations et des spécificités des 100 milliards de neurones. Si les neurones peuvent se caractériser selon des critères structuraux (taille, nombre de neurites) et fonctionnels (neurotransmetteurs synthétisés et type de connexions), leur contribution est spécifique de la zone cérébrale.

Par ailleurs, I'organisation synaptique ne peut plus être considérée sans prendre en compte un autre partenaire cellulaire, l'astrocyte dont les longs prolongements enrobent d'un côté les capillaires cérébraux et de l'autre la synapse assurant ainsi un couplage entre l'activité neuronale et la demande énergétique (figure 1). La plasticité de ces relations neurone-astrocytescellules endothéliales tient essentiellement à la nature de la cellule astrocytaire dont on a pu montrer la capacité de transformation morphologique importante. Cette propriété per- met sous l'influence de facteurs externes (tels que les neurotransmetteurs) d'émettre ou de rétracter de fins prolongements, de s'organiser en réseau communicant ou de s'isoler. En modulant l'environnement extracellulaire, les astrocytes peuvent simultanément modifier la sensibilité d'un grand nombre de neurones et jouer ainsi sur la coordination fonctionnelle des cellules nerveuses. Ces observations ont pu être faites in situ dans 2 systèmes différents de I'hypothalamus, les noyaux supraoptiques répondant à des variations saisonnières [24] et les noyaux suprachiasmatiques, siège de l'horloge circadienne, répondant à des variations journalières [25]. Ces manifestations physiologiques qui permettent à l'organisme de s'adapter aux variations de l'environnement deman- 


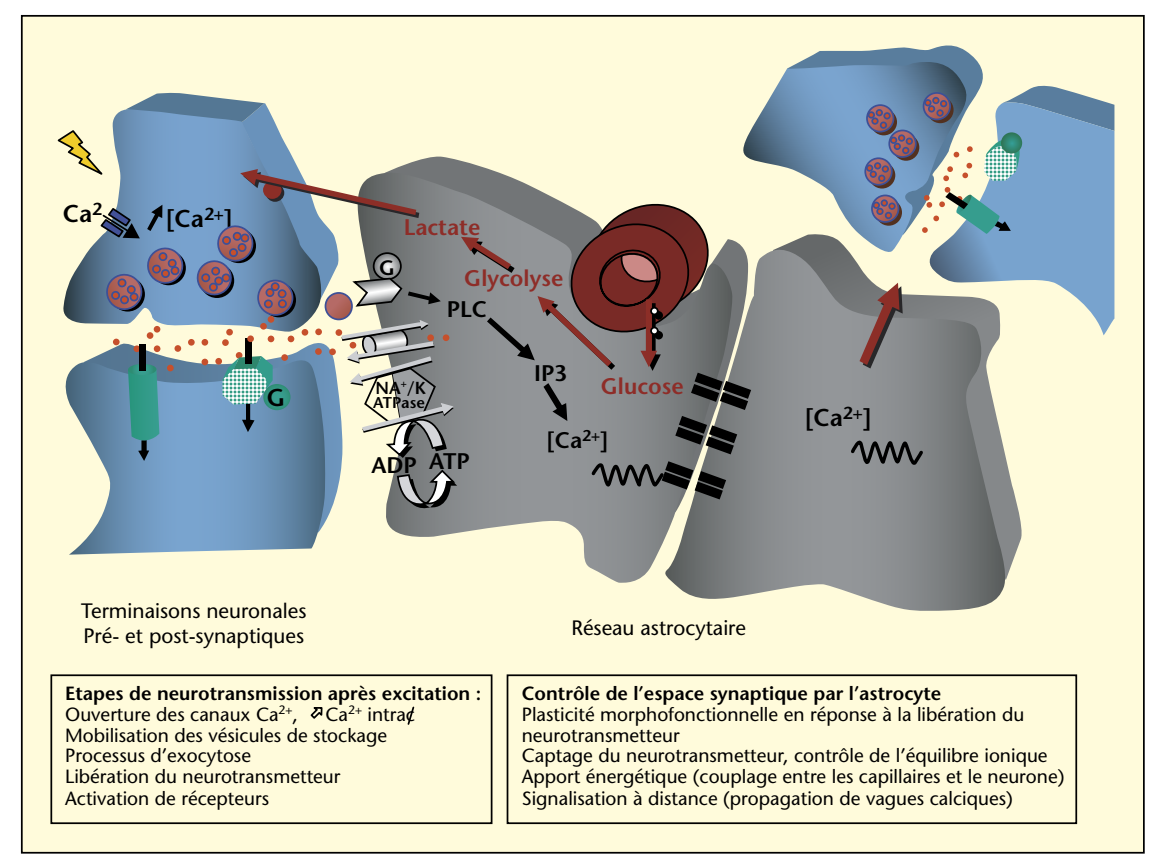

Figure 1. Le complexe synaptique.

dent une grande plasticité qui se manifeste notamment par des réorganisations membranaires. Tous les processus mettant en jeu les protéines transmembranaires (canaux, récepteurs et transporteurs), la fusion membranaire entre vésicules de stockage et membrane plasmique à la terminaison présynaptique (pour l'exocytose), la motilité cellulaire seront autant de cibles des AGPI et notamment des n-3.

Par ailleurs, dans le complexe synaptique les AGPI peuvent intervenir lors d'une activation dépolarisante. Le neurotransmetteur libéré, en se liant aux récepteurs, agira indirectement sur la libération des AGPI via notamment l'activation de la phospholipase A2. À ce niveau, leur action est essentiellement identifiée au travers de celle de l'AA ou de son dérivé oxygéné la prostaglandine E2 pour lesquels il a été décrit des rôles multiples sur les récepteurs et transporteurs neuronaux et astrocytaires, sur la propagation des vagues calciques, sur la vasodilatation des capillaires cérébraux [26]. Un certain nombre de données récentes implique aussi le DHA dans la régulation synaptique. II a été montré que le DHA augmente la résistance à la toxicité au glutamate [27], protège contre la mort neuronale induite par le kaïnate ou I'ischémie [28], favorise la survie neuronale [29, 30]. Ces observations sont à relier à celles de Berry et McBean [31] qui démontrent une augmentation du captage de l'aspartate en présence de DHA. Nous avons par ailleurs observé une amélioration de la communication astrocytaire via les jonctions communicantes lorsque le milieu de culture est supplémenté avec $30 \mu \mathrm{M}$ de DHA [32]. La réduction décrite de
I'activité Na K-ATPase [33] et Ca ATPase [34] est également à prendre en compte dans la compréhension du rôle joué par les AGPI au niveau synaptique.

Les dernières conceptions de la neurotransmisment rapide se déplaçant latéralement vers et hors de la synapse. Lors d'une activation, l'augmentation $\mathrm{du} \mathrm{Ca}{ }^{2+}$ intracellulaire déclenche une immobilisation des récepteurs et une accumulation locale à la surface neuronale [35]. Sur ces bases, on est en droit de se poser la question de l'importance de la teneur en DHA dans les membranes pour favoriser les déplacements rapides à la surface des neurones postsynaptiques. Certains de ces récepteurs étant ancrés dans les radeaux lipidiques, l'incidence de la variation de la teneur en DHA sur la composition de ces radeaux est aussi à étudier.

Pour conclure, une question reste à éclairer, celle de la mise en place des structures et de la synaptogenèse. Des expériences in vitro et in vivo établissent que le DHA accélère la différenciation neuronale, accélère la pousse neuritique et la maturation des neurones différenciés, et favorise la neurogenèse chez le rat adulte $[30,36,37]$. Des travaux très récents [38] mettent en évidence une altération de la morphologie et de l'organisation des structures télencéphaliques chez l'embryon de rat E19 issus de mère déficientes en AGPn-3.

L'ensemble de ces références mettent en avant I'ampleur de l'action possible des AGPn-3 et du DHA au niveau synaptique et ne peuvent que sion mettent en jeu des récepteurs en mouve- nous encourager à approfondir ces connaissances pour mieux déterminer les besoins essentiels au bon fonctionnement cérébral.

\section{RÉFÉRENCES}

1. DURAND G, GUESNET P, CHALON S, ALESSANDRI JM, RIZKALLA S, LEBRANCHUY. Importance nutrionnelle des acides gras polyinsaturés. In : Roberfroid M, ed. Aliments Fonctionnels. Paris: Editions Tec \& Doc-Lavoisier, 2002 : 193-219.

2. GUESNET P, ALESSANDRI JM. Acides gras polyinsaturés du lait et développment du système nerveux central du nouveau-né. Cah Nutr Diét $1995 ; 30: 109-11$.

3. GUESNET P, ALASNIER C, ALESSANDRI JM, DURAND G. Modifying the $\mathrm{n}-3$ fatty acid content of the maternal diet to determine the requirements of the feta land suckling rat. Lipids $1997 ; 32: 527-34$.

4. BOURRE JM, FRANCOIS M, YOUYOU A, et al. The effects of dietary alpha-linolenic acid on the composition of nerve membranes, enzymatic activity, amplitude of electrophysiological parameters, resistance to poisons and performance of learning tasks in rats. / Nutr 1989 ; $119: 1880-92$.

5. GREINER RS, MORIGUCHI T, HUTTON A, SLOTNICK BM, SALEM JR. N. Rats with low levels of brain docosahexaenoic acid show impaired performance in olfactory-based and spatial learning tasks. Lipids 1999 ; 34 : S239-S243.

6. MORIGUCHIT, GREINER RS, SALEM IR. N. Behavioral deficits associated with dietary induction of decreased brain docosahexaenoic acid concentration. / Neurochem 2000; 75 : 2563-73.

7. MORIGUCHI T, SALEM IR. N. Recovery of brain docosahexaenoate leads to recovery of spatial task performance. / Neurochem 2003; 87 . 297-309.

8. LIM SY, HOSHIBA J, MORIGUCHI T, SALEM JR. N. $\mathrm{N}-3$ fatty acid deficiency induced by a modified artificial rearing method leads to poorer performance in spatial learning tasks. Pediatr Res 2005 ; $58: 741-8$.

9. UMEZAWA M, OHTAA, TOJO H, YAGI $\mathrm{H}$, HOSOKAWAM, TAKEDAT. Dietary alphalinolenate/linoleate balance influences learning and memory in the senescence-accelerated mouse (SAM). Brain Res $1995 ; 669$ : 225-33.

10. WAINWRIGHT PE, HUANG YS, BULMANFLEMING B, LEVESQUE S, MCCUTCHEON D. The effects of dietary fatty acid composition combined with environmental enrichment on brain and behavior in mice. Behav Brain Res $1994 ; 60: 125-36$.

11. VANCASSEL S, AID S, PIFFERI F, et al. Cerebral asymmetry and behavioral lateralization in rats chronically lacking $n-3$ polyunsaturated fatty acids. Biol Psychiatry $2005 ; 58$ : 805-11. 
12. XIMENES DA SILVAA, LAVIALLE F, GENDROT G, GUESNET P, ALESSANDRI JM, LAVIALLE M. Glucose transport and utilization are altered in the brain of rats deficient in $\mathrm{n}-3$ polyunsaturated fatty acids. I Neurochem $2002 ; 81: 1328-37$.

13. PIFFERI F, ROUX F, LANGELIER $B$, et al. (n-3) polyunsaturated fatty acid deficiency reduces the expression of both isoforms of the brain glucose transporter GLUT1 in rats. J Nutr 2005 ; $135: 2241-6$.

14. CHALON S. Omega-3 fatty acids and monoamine neurotransmission. Prostaglandins Leukot Essent Fatty Acids 2006 ; 75 : 259-69 ; (revue).

15. YOSHIDA S, YASUDA A, KAWAZATO $H$, et al. Synaptic vesicle ultrastructural changes in the rat hippocampus induced by a combination of alpha-linolenate deficiency and a learning task. J Neurochem 1997 ; 68 : 1261-8.

16. INNIS SM, DE LA PRESA OWENSS. Dietary fatty acid composition in pregnancy alters neurite membrane fatty acids and dopamine in newborn rat brain. / Nutr $2001 ; 131$ : 118-22.

17. DELION S, CHALON S, HERAULT J, GUILLOTEAU D, BESNARD JC, DURAND G. Chronic dietary alpha-linolenic acid deficiency alters dopaminergic and serotoninergic neurotransmission in rats. J Nutr $1994 ; 124: 2466-76$.

18. KODAS E, GALINEAU L, BODARD S, et al. Serotoninergic neurotransmission is affected by $n-3$ polyunsaturated fatty acids in the rat. / Neurochem $2004 ; 89: 695-702$.

19. CHALON S, DELION-VANCASSEL S, BELZUNG C et al. Dietary fish oil affects monoaminergic neurotransmission and behavior in rats. J Nutr 1998 ; $128: 2512-9$.

20. DE LA PRESA OWENSS, INNIS SM. Docosahexaenoic and arachidonic acid prevent a decrease in dopaminergic and serotoninergic neurotransmitters in frontal cortex caused by a linoleic and alpha-linolenic acid deficient diet in formula-fed piglets. / Nutr $1999 ; 129$ : 208893.
21. KODAS E, VANCASSEL S, LEJEUNE B, GUILLOTEAU D, CHALON S. Reversibility of $n-3$ fatty acid deficiency-induced changes in dopaminergic neurotransmission in rats : critical role of developmental stage. / Lipid Res 2002; 43 : 1209-19.

22. AID $S$, VANCASSEL $S$, POUMES-BALLIHAUT $C$, CHALON S, GUESNET P, LAVIALLE M. Effect of a diet-induced n-3 PUFA depletion on cholinergic parameters in the rat hippocampus. / Lipid Res $2003 ; 44$ : 1545-51.

23. AID S, VANCASSEL $S$, LINARD A, LAVIALLE M, GUESNET P. Dietary docosahexaenoic acid [22:6(n-3)] as a phospholipid or a triglyceride enhances the potassium chloride-evoked release of acetylcholine in rat hippocampus. I Nutr $2005 ; 135: 1008-13$

24. THEODOSIS DT, POULAIN DA. Activity-dependent neuronal-glial and synaptic plasticity in the adult mammalian hypothalamus. Neuroscience $1993 ; 57: 501-35$.

25. LAVIALLE M, BEGUE A, PAPILLON C, VILAPLANA J. Modifications of retinal afferent activity induce changes in astroglial plasticity in the hamster circadian clock. Glia 2001; 34 : 88-100.

26. ALESSANDRI JM, GUESNET P, VANCASSEL $S$, et al. Polyunsaturated fatty acids in the central nervous system : evolution of concepts and nutritional implications throughout life. Reprod Nutr Dev 2004 ; 44 : 509-38.

27. WANG $X$, ZHAOX, MAO ZY, WANG XM, LIU ZL. Neuroprotective effect of docosahexaenoic acid on glutamate-induced cytotoxicity in rat hippocampal cultures. Neuroreport 2003; $14: 2457-61$

28. LAURITZEN I, BLONDEAU N, HEURTEAUX C, WIDMANN C, ROMEY G, LAZDUNSKI M. Polyunsaturated fatty acids are potent neuroprotectors. EMBO / $2000 ; 19$ : 1784-93.
29. HOGYES E, NYAKAS C, KILIAAN A, FARKAS T, PENKE B, LUITEN PG. Neuroprotective effect of developmental docosahexaenoic acid supplement against excitotoxic brain damage in infant rats. Neuroscience 2003; 119: 9991012.

30. CAO D, XUE R, XU J, LIU Z. Effects of docosahexaenoic acid on the survival and neurite outgrowth of rat cortical neurons in primary cultures. J Nutr Biochem 2005 ; 16 : 538-46.

31. BERRY (B, MCBEAN G). An investigation into the role of calcium in the modulation of rat synaptosomal D-[3H]aspartate transport by docosahexaenoic acid. Brain Res 2003 ; 973 : 107-14.

32. CHAMPEIL-POTOKAR G, CHAUMONTET C, GUESNET P, LAVIALLE M, DENIS I. Docosahexaenoic acid (22: 6n-3) enrichment of membrane phospholipids increases gap junction coupling capacity in cultured astrocytes. Eur / Neurosci 2006 ; 24 : 3084-90.

33. GERBI A, ZEROUGA M, MAIXENT JM, DEBRAY M, DURAND G, BOURRE JM. Diet deficient in alpha-linolenic acid alters fatty acid composition and enzymatic properties of $\mathrm{Na}+$, $\mathrm{K}+$-ATPase isoenzymes of brain membranes in the adult rat. / Nutr Biochem 1999 ; 10 : 30-6.

34. KEARNS SD, HAAG M. The effect of omega-3 fatty acids on Ca-ATPase in rat cerebral cortex. Prostaglandins Leukot Essent Fatty Acids 2002 ; $67: 303-8$.

35. BORGDORFF Al, CHOQUET D. Regulation of AMPA receptor lateral movements. Nature $2002 ; 417: 649-53$.

36. CALDERON F, KIM HY. Docosahexaenoic acid promotes neurite growth in hippocampal neurons. J Neurochem 2004 ; 90 : 979-88.

37. KAWAKITAE, HASHIMOTO M, SHIDO O. Docosahexaenoic acid promotes neurogenesis in vitro and in vivo. Neuroscience $2006 ; 139$ : 991-7.

38. COTI BERTRAND P, O'KUSKY JR, INNIS SM. Maternal dietary $(n-3)$ fatty acid deficiency alters neurogenesis in the embryonic rat brain. I Nutr 2006 ; 136 : 1570-5. 